\title{
Line-of-sight velocity distributions of 53 early-type galaxies ${ }^{\star}$
}

\author{
W. Koprolin and W.W. Zeilinger \\ Institut für Astronomie der Universität Wien, Türkenschanzstraße 17, A-1180 Wien, Austria
}

Received December 1, 1999; accepted April 20, 2000

\begin{abstract}
. 55 long-slit spectra of 53 early-type galaxies were observed at La Silla/ESO and reduced using standard methods. The line-of-sight velocity distributions (LOSVDs) were measured using the fourier quotient method and the fourier fitting method as described by van der Marel et al. (1993). 32\% of the examined galaxies contain kinematically decoupled stellar omponents, the size of these cores was $0.40 \pm 0.28 \mathrm{kpc}$, in each case the core was smaller than $1 \mathrm{kpc}$. Analysis of the kinematics reveals in $49 \%$ of the sample galaxies the signature of a stellar disk component, in $15 \%$ this is uncertain. There is evidence that the phenomenon of kinematically decoupled components is present in the whole class of early-type galaxies. Several correlations between photometric and kinematic parameters like the $(v / \sigma)^{*}$ vs. $\epsilon$ diagram, the anisotropy - luminosity correlation or $\kappa$-space were as well examined using measurement results for spectroscopic data and photometric data out of literature. It is also shown that those sample galaxies with kinematically decoupled components are more likely to be found in groups of high density, strengthening the assumption that such components are remnants of merging events.
\end{abstract}

Key words: galaxies: elliptical and lenticular galaxies: fundamental parameters — galaxies: kinematics and dynamics

\section{Introduction}

The traditional picture of describing elliptical galaxies as simple isothermal bodies which are chemically well mixed has dramatically changed. Ellipticals are now seen as complex systems from the photometrical, kinematical and chemical point of view. The properties of ellipticals are found to correlate with their mass (Burstein et al. 1997).

Send offprint requests to: W. Koprolin

* Full Fig. 6 is only available at http://www.edpsciences.org
Large ellipticals are triaxial systems supported by anisotropic velocity distributions of the stars. Low-luminosity systems are on the contrary generally axisymmetric rotationally supported bodies.

One of the most noteable features observed in ellipticals are kinematically decoupled core components. The most extreme cases are peculiar cores which are characterized by angular momentum vectors which are opposite or perpendicular with respect to the main body of the galaxy. Merging seems to be a plausible explanation for the origin of the decoupled component. This is also supported by the fact that the metallicities of the core component as measured through the absorption line indices appear to be enhanced with respect to the rest of the galaxy (Bender \& Surma 1992). In about $30 \%$ of the nearby luminous ellipticals peculiar core kinematics are detected. Taking however projection effects into account, it is estimated that more than $50 \%$ of all luminous ellipticals should contain a kinematically decoupled core (Bender 1996).

In order to study the stellar kinematical properties of ellipticals in a homogenous manner, we carried out a spectroscopic study of a sample of 53 southern ellipticals. In the following section we summarize the observations and data reduction. In Sect. 3 we present the results obtained by application of the fourier fitting method as described by van der Marel \& Franx (1993). The correlations between global photometric and kinematic properties are discussed in Sect. 4. Finally we discuss some conclusion on the merging history of the class of elliptical galaxies which can be drawn on the basis of the sample.

\section{Observations and data reduction}

In alltogether 15 nights 55 long-slit spectra of 53 earlytype galaxies were obtained using the ESO $1.52 \mathrm{~m}$ telescope equipped with a B\&C Cassegrain spectrograph. The observation $\log$ is given in Table 2. The parameters of the instrumental setups are described in Table 3. Instrumental dispersions for the different setups were determined by measuring the width of night-sky emission 
lines and helium-argon lines in the calibration spectra. The seeing was typically $1.5-2$ arcsec. The detectors employed were all Ford Aerospace $2048 \times 2048$ pixel CCDs with a pixel size of $15 \times 15 \mu \mathrm{m}^{2}$. The position angle of the slit was generally aligned with the optical major axis of the target galaxy.

The integration times range from 40 to $120 \mathrm{~min}$. The observed sample contains little less than half of all earlytype galaxies with systemic velocities $<3000 \mathrm{~km} \mathrm{~s}^{-1}$. Exceptions are NGC $3302\left(v_{\text {sys }}=4075 \mathrm{~km} \mathrm{~s}^{-1}\right)$ and NGC $3309\left(v_{\text {sys }}=4057 \mathrm{~km} \mathrm{~s}^{-1}\right)$. Table 1 lists the sample galaxies and their basic parameters.

The spectra were calibrated with the ESO images processing package MIDAS using standard procedures for bias subtraction and flat field correction. Artifacts produced by cosmic ray events were removed by applying a filtering algorithm and by visual inspection. The science spectra were bracketed by helium-argon calibration spectra in order to verify shifts along the dispersion due to instrumental flexure. No significant shifts were however detected. Wavelength calibration was performed on the science frames for each spectral row independently by fitting a third order polynomial, using the respective averaged helium-argon spectra as reference. The rms error of the dispersion curve was typically $<0.4 \AA$. A sky spectrum was derived for each galaxy spectrum by fitting each wavelength bin with a first order polynomial at the border regions of the frame uncontaminated by the contribution of the galaxy light. To obtain an acceptable $S / N$ all over the spectra, several lines in spatial direction were averaged until $S / N>12$ was reached, where necessary. Within the central $3 \operatorname{arcsec}$ of the galaxies $S / N \approx 35$ is typical.

For the subsequent analysis of the stellar kinematics the fourier fitting method using Gauss-Hermite polynomials was used as described by van der Marel et al. (1993). Spectra of non-rotating $\mathrm{K}$ and $\mathrm{M}$ giants obtained in the same instrumental setup as the galaxy spectra were used as velocity templates. Typically 4 to 8 such template stars were observed in each observing run.

\section{Results}

Emission lines were detected in 5 galaxies of the sample: UGC 4508, NGC 1889, NGC 3125, NGC 5237 and UGC 7354. Table 4 lists the detected emission lines of the respective galaxies and their properties. Analysis suggests photoionisation to be the main cause for the line emission, which is typical for H II regions.

The results for the stellar kinematics obtained for all 49 sample galaxies with absorption lines strong enough for fourier fitting are presented in the Appendix. The radial velocity and velocity dispersion profiles compare favorably with previously published measurements (Bender et al. 1994; Davies et al. 1983; Franx et al. 1989; Binney et al. 1990; Carollo \& Danziger 1994a). The agreement of the kinematical profiles is for the major part within $1 \sigma$ deviation. For most of the galaxies of our sample no published $h_{3}$ and $h_{4}$ are available for comparison.

\section{Notes on individual objects:}

IC 1729: Asymmetric radial velocity profile, central depression in the velocity dispersion profile.

NGC 1404: Disk without rotation within $0.1 r_{\mathrm{e}}$, velocity profile constant within $1 r_{\mathrm{e}}, h_{3} \propto-v$ : typical example of decoupled central component kinematics.

UGC 4508: cE? High-intensity emission lines, low continuum.

NGC 1889: In interaction with the disklike galaxy NGC 1888, merger. The kinematic profiles overlap, resulting in a velocity dispersion profile and line strength increase outward. $\mathrm{H} \alpha$ and $[\mathrm{NII}]$ line emission were detected, possibly from the neighboring galaxy. Liu \& Kennicutt (1995) state NGC 1888 to be a spiral of the type $\mathrm{Sa}$ or $\mathrm{Sb}$ with dominent old stellar population and only weak line emission.

NGC 2663: Very small rotation, unusually high velocity dispersion. This galaxy is a massive brightest cluster member and characterized by very anisotropic dynamics (Carollo \& Danziger 1994b). The galaxy also features a compact central radio source (Danziger \& Goss 1983; Sadler et al. 1989).

NGC 2271: Kinematically decoupled central component within $1.5^{\prime \prime}$ of the galaxy's center without rotation.

NGC 3078: Slightly asymmetric kinematically decoupled central component without rotation, also supported by a central depression in the $\mathrm{Mg}_{2}$ profile (Carollo et al. 1993). NGC 3125 = AM 1004-294: High intensity emission lines, low continuum. This is a bright, compact low-redshift galaxy in a cluster with regular outer isophotes and double knots, it is classified as an amorphous $\mathrm{H}$ II galaxy (Telles et al. 1997) or a blue compact dwarf (Marlowe et al. 1997).

NGC 3136: Counter-rotation disk $4^{\prime \prime}$ away from the center of intensity with a diameter of $2^{\prime \prime}$; significance is low.

NGC 3250: Counter-rotating disk $9^{\prime \prime}$ away from the center of intensity with a diameter of $3^{\prime \prime}, h_{3} \propto-v$, indicating decoupled central component kinematics (Bender et al. 1994). The $\mathrm{Mg}_{2}$ profile of this galaxy also features a central depression (Carollo et al. 1993).

NGC 3268: This galaxy features rotation only at one side of the core probably due to the presence of dust. It also has a parsec-scale radio core (Slee et al. 1994). $h_{3} \propto v$, indicating a disk component (Bender et al. 1994).

NGC 3302: Dust lane along major axis.

NGC 3557B: Possible kinematically decoupled central component supported by the asymmetric rotational velocity profile and $h_{3}>0$, low significance because of low spatial resolution.

NGC 3636: E0 with low velocity dispersion $\left(\sigma<160 \mathrm{~km} \mathrm{~s}^{-1}\right)$. 
Table 1. Programme galaxies and their basic parameters

\begin{tabular}{|c|c|c|c|c|c|c|c|}
\hline $\begin{array}{l}\text { Object } \\
\left(\mathrm{PGC}^{1}\right) \\
\end{array}$ & $\begin{array}{c}\text { Object } \\
(\mathrm{NGC} / \mathrm{IC} / \mathrm{UGC}) \\
\end{array}$ & $\begin{array}{c}\text { Type } \\
\left(\mathrm{RC} 3^{2}\right) \\
\end{array}$ & $\begin{array}{c}\text { Type } \\
\left(\mathrm{RSA}^{3}\right) \\
\end{array}$ & $\begin{array}{l}\text { Type } \\
\text { (PGC) }\end{array}$ & $\begin{array}{l}B_{\mathrm{T}}^{0} \\
(\mathrm{ESC} \\
\end{array}$ & $\begin{array}{c}A_{\mathrm{e}} \\
\text {-Uppsa }\end{array}$ & $\begin{array}{l}V_{\text {hel }} \\
\left.\operatorname{lat}^{4}\right)\end{array}$ \\
\hline 6588 & IC 1729 & $\overline{\mathrm{SABO}^{-}}$: & & $\overline{\mathrm{E}}$ & 13.28 & 29.8 & 1495 \\
\hline 13433 & NGC 1404 & E1 & E2 & $\mathrm{E}$ & 10.89 & 26.7 & 1925 \\
\hline 13609 & NGC 1427 & $\mathrm{cD}$ & E5 & $\mathrm{E}$ & 11.76 & 32.9 & 1430 \\
\hline 14695 & NGC 1537 & $\mathrm{SAB}^{-}$pec? & E6 & LB & 11.53 & 26.1 & 1371 \\
\hline 14757 & NGC 1549 & E0 & $\mathrm{E} 2$ & $\mathrm{E}$ & 10.58 & 47.5 & 1153 \\
\hline 17196 & NGC 1889 & cD pec: & & E M & 14.1 & & 2472 \\
\hline 19476 & NGC 2271 & $\mathrm{SABO}^{-}$ & & $\mathrm{LB}$ & 12.11 & 64.3 & 2588 \\
\hline 20047 & NGC 2325 & E4 & E4 & $\mathrm{E}$ & 10.96 & 86.5 & 2248 \\
\hline 20916 & NGC 2380 & SAB0: & & LB & 10.38 & 43.4 & 1775 \\
\hline 21325 & NGC 2434 & E0 & E0 & $\mathrm{E}$ & 11.43 & 40.5 & 1327 \\
\hline 23304 & IC 2311 & E0: & & $\mathrm{E}$ & 11.00 & 17.7 & 1836 \\
\hline 24352 & UGC 4508 & $\mathrm{cE} ?$ & & $\mathrm{~S}$ & 14.90 & & 1917 \\
\hline 24590 & NGC 2663 & $\mathrm{E}$ & & $\mathrm{E}$ & 10.23 & 84.6 & 2048 \\
\hline 25075 & NGC 2699 & E: & & $\mathrm{E}$ & 13.57 & & 1825 \\
\hline 26592 & NGC 2887 & $\mathrm{SA} 0(\mathrm{~s})^{-} ?$ & & $\mathrm{~L}$ & 11.72 & 27.4 & 2850 \\
\hline 26601 & NGC 2865 & E3 & E4 & $\mathrm{E}$ & 12.29 & 11.7 & 2581 \\
\hline 26733 & NGC 2872 & $\mathrm{E} 2$ & & $\mathrm{E}$ & 12.64 & 20.8 & 3226 \\
\hline 26768 & NGC 2888 & $\mathrm{cD}:$ & E2 & $\mathrm{E}$ & 12.98 & 14.7 & 2233 \\
\hline 27885 & NGC 2986 & $\mathrm{E} 2$ & $\mathrm{E} 2$ & $\mathrm{E}$ & 11.51 & 41.4 & 2264 \\
\hline 28806 & NGC 3078 & E2 & E3 & $\mathrm{E}$ & 11.93 & 23.8 & 2506 \\
\hline 28845 & NGC 3087 & $\mathrm{cD}:$ & $\mathrm{E} 2$ & $\mathrm{E}$ & 12.34 & 16.1 & 2662 \\
\hline 29311 & NGC 3136 & E: & E4 & $\mathrm{E}$ & 11.23 & 41.4 & 1647 \\
\hline 29366 & NGC 3125 & $\mathrm{E} ?$ & Amorphous & $\mathrm{E}$ & 13.16 & 9.2 & 1080 \\
\hline 30314 & NGC 3224 & $\mathrm{cD}$ & & $\mathrm{E}$ & 12.48 & 15.3 & 3088 \\
\hline 30671 & NGC 3250 & $\mathrm{E} 4$ & E3 & $\mathrm{E}$ & 11.67 & 32.1 & 2883 \\
\hline 30859 & NGC 3258 & E1 & E1 & $\mathrm{E}$ & 12.27 & 27.4 & 2808 \\
\hline 30875 & NGC 3260 & E pec: & & $\mathrm{E}$ & 13.06 & 25.0 & 2413 \\
\hline 30949 & NGC 3268 & E2 & E2 & $\mathrm{E}$ & 12.23 & 36.1 & 2761 \\
\hline 31391 & NGC 3302 & $\mathrm{SA} 0$ & & $\mathrm{~L}$ & 13.16 & 20.4 & 4075 \\
\hline 31466 & NGC 3309 & E3 & E1 & $\mathrm{E}$ & 12.34 & 31.4 & 4057 \\
\hline 32249 & NGC 3377 & E5 & E6 & $\mathrm{E}$ & 11.13 & 33.7 & 689 \\
\hline 33824 & NGC 3557B & E5: & & E M & 12.92 & & 2855 \\
\hline 33871 & NGC 3557 & E3 & E3 & $\mathrm{E}$ & 11.13 & 37.8 & 3014 \\
\hline 34160 & NGC 3585 & E7 & $\mathrm{E} 7 / \mathrm{SO}_{1}(7)$ & $\mathrm{E}$ & 10.53 & 39.5 & 1491 \\
\hline 24513 & NGC 3617 & $\mathrm{cD}$ & & & 13.26 & 11.9 & 1491 \\
\hline 34709 & NGC 3636 & E0 & & $\mathrm{E}$ & 13.19 & & 1715 \\
\hline 36918 & NGC 3904 & E2: & E2 & $\mathrm{E}$ & 11.67 & 23.3 & 1750 \\
\hline 37061 & NGC 3923 & $\mathrm{E} 4$ & $\mathrm{E} 4 / \mathrm{S}_{1}(4)$ & $\mathrm{E}$ & 10.52 & 53.3 & 1607 \\
\hline 37863 & NGC 4033 & E6 & $\mathrm{S}_{1}(6)$ & $\mathrm{E}$ & 12.52 & 16.1 & 1521 \\
\hline 38411 & NGC 4105 & E3 & $\mathrm{S}_{1 / 2}(3)$ & E M & 11.21 & 41.4 & 1900 \\
\hline 38417 & NGC 4106 & $\mathrm{SBO}(\mathrm{s})^{+}$ & SB0/a (tides) & LB & 11.88 & 61.0 & 2189 \\
\hline 39628 & UGC 7354 & & & $\mathrm{E}$ & 14.66 & & 1684 \\
\hline 39659 & NGC 4261 & E2 & E3 & $\mathrm{E}$ & 11.32 & 28.6 & 2200 \\
\hline 43276 & NGC 4697 & E6 & E6 & $\mathrm{E}$ & 10.03 & 75.4 & 1210 \\
\hline 46330 & NGC 5061 & E0 & E0 & $\mathrm{E}$ & 11.06 & 25.5 & 2065 \\
\hline 48139 & NGC 5237 & I0? & & E M & 12.84 & 26.9 & 321 \\
\hline 54646 & NGC 5903 & E2 & $\mathrm{E} 3 / \mathrm{S}_{1}(3)$ & $\mathrm{E}$ & 11.76 & 35.2 & 2519 \\
\hline 62589 & IC 4797 & cD pec: & $\mathrm{E} 5 / \mathrm{S}_{1}(5)$ & $\mathrm{E}$ & 12.04 & 17.7 & 2582 \\
\hline 64136 & NGC 6861 & $\mathrm{SA} 0(\mathrm{~s})^{-}:$ & $\mathrm{S}_{3}(6)$ & LB & 11.78 & 22.8 & 2819 \\
\hline 66318 & NGC 7029 & E6: & $\mathrm{S}_{1}(5)$ & $\mathrm{E}$ & 12.26 & 24.4 & 2818 \\
\hline 66549 & NGC 7049 & $\mathrm{SA}(\mathrm{s}) 0$ & $\mathrm{SO}_{3}(4) / \mathrm{Sa}$ & $\mathrm{L}$ & 11.42 & 47.8 & 2198 \\
\hline 68020 & NGC 7196 & E: & $\mathrm{E} 3 / \mathrm{SO}_{3}(3)$ & $\mathrm{E}$ & 12.31 & 21.7 & 3007 \\
\hline 70975 & IC 5297 & & & $\mathrm{~S}$ & 15.6 & & \\
\hline
\end{tabular}

1 Paturel et al. 1989

2 de Vaucouleurs et al. 1991.

${ }^{3}$ Sandage \& Tammann 1981.

${ }^{4}$ Lauberts \& Valentijn 1989. 
Table 2. Observation log

\begin{tabular}{|c|c|c|c|c|}
\hline $\begin{array}{c}\text { observing } \\
\text { run }\end{array}$ & date & galaxy & $\begin{array}{c}\text { integration } \\
\text { time }\end{array}$ & $\begin{array}{c}\text { position } \\
\text { angle }\end{array}$ \\
\hline \multirow[t]{5}{*}{191991} & Mar. 24/25 & NGC 3302 & $7200 \mathrm{~s}$ & $28^{\circ}$ \\
\hline & & NGC 4697 & $3600 \mathrm{~s}$ & $80^{\circ}$ \\
\hline & Mar. 25/26 & NGC 3309 & $7200 \mathrm{~s}$ & $170^{\circ}$ \\
\hline & Mar. 26/27 & NGC 4105 & $7200 \mathrm{~s}$ & $115^{\circ}$ \\
\hline & & NGC 4106 & $7200 \mathrm{~s}$ & $115^{\circ}$ \\
\hline \multirow[t]{21}{*}{1992} & Jan. 29/30 & NGC 1537 & $2 \times 2400 \mathrm{~s}$ & $94^{\circ}$ \\
\hline & & NGC 2325 & $2 \times 2400 \mathrm{~s}$ & $6^{\circ}$ \\
\hline & & NGC 2663 & $2 \times 2400 \mathrm{~s}$ & $113^{\circ}$ \\
\hline & & NGC 3078 & $2400 \mathrm{~s}$ & $15^{\circ}$ \\
\hline & & NGC 3268 & $2400 \mathrm{~s}$ & $62^{\circ}$ \\
\hline & Jan. $30 / 31$ & NGC 2271 & $2 \times 2400 \mathrm{~s}$ & $91^{\circ}$ \\
\hline & & NGC 2865 & $2 \times 2400 \mathrm{~s}$ & $128^{\circ}$ \\
\hline & & NGC 3087 & $2 \times 2400 \mathrm{~s}$ & $126^{\circ}$ \\
\hline & & NGC 3136 & $2 \times 2400 \mathrm{~s}$ & $126^{\circ}$ \\
\hline & Jan. 31/Feb. 1 & NGC 1549 & $2 \times 2400 \mathrm{~s}$ & $4^{\circ}$ \\
\hline & & NGC 3250 & $2 \times 2400 \mathrm{~s}$ & $136^{\circ}$ \\
\hline & & NGC 3258 & $2 \times 2400 \mathrm{~s}$ & $55^{\circ}$ \\
\hline & Feb. 1/2 & NGC 1427 & $2 \times 2400 \mathrm{~s}$ & $77^{\circ}$ \\
\hline & & NGC 2380 & $2 \times 2400 \mathrm{~s}$ & $72^{\circ}$ \\
\hline & & NGC 3224 & $2 \times 2400 \mathrm{~s}$ & $134^{\circ}$ \\
\hline & & NGC 3585 & $2 \times 2400 \mathrm{~s}$ & $96^{\circ}$ \\
\hline & & NGC 3904 & $2400 \mathrm{~s}, 1200 \mathrm{~s}$ & $0^{\circ}$ \\
\hline & Feb. $2 / 3$ & NGC 2888 & $2 \times 2400 \mathrm{~s}$ & $170^{\circ}$ \\
\hline & & NGC 2434 & $2 \times 2400 \mathrm{~s}$ & $126^{\circ}$ \\
\hline & & NGC 2887 & $2 \times 2400 \mathrm{~s}$ & $87^{\circ}$ \\
\hline & & NGC 5061 & $1800 \mathrm{~s}, 1500 \mathrm{~s}$ & $95^{\circ}$ \\
\hline \multirow[t]{8}{*}{1992} & Aug. 25/26 & NGC 5903 & $2 \times 2700 \mathrm{~s}$ & $139^{\circ}$ \\
\hline & & IC 4797 & $2 \times 2700 \mathrm{~s}$ & $145^{\circ}$ \\
\hline & & NGC 7029 & $2 \times 2700 \mathrm{~s}$ & $69^{\circ}$ \\
\hline & & NGC 7196 & $2 \times 2700 \mathrm{~s}$ & $47^{\circ}$ \\
\hline & & IC 1729 & $2700 \mathrm{~s}$ & $142^{\circ}$ \\
\hline & & NGC 1404 & $2700 \mathrm{~s}$ & $160^{\circ}$ \\
\hline & Aug. $26 / 27$ & NGC 7049 & $2 \times 3600 \mathrm{~s}$ & $57^{\circ}$ \\
\hline & Aug. $28 / 29$ & NGC 6861 & $3600 \mathrm{~s}$ & $140^{\circ}$ \\
\hline \multirow[t]{21}{*}{1993} & Feb. 16/17 & IC 2311 & $3600 \mathrm{~s}$ & $84^{\circ}$ \\
\hline & & NGC 2699 & $3600 \mathrm{~s}$ & $50^{\circ}$ \\
\hline & & NGC 2986 & $3600 \mathrm{~s}$ & $78^{\circ}$ \\
\hline & & NGC 3377 & $3600 \mathrm{~s}$ & $36^{\circ}$ \\
\hline & & NGC 3923 & $3600 \mathrm{~s}$ & $50^{\circ}$ \\
\hline & Feb. $17 / 18$ & NGC 1889 & $3600 \mathrm{~s}$ & $161^{\circ}$ \\
\hline & & NGC 3125 & $3600 \mathrm{~s}$ & $111^{\circ}$ \\
\hline & & IC 5297 & $3600 \mathrm{~s}$ & $0^{\circ}$ \\
\hline & & NGC 3617 & $3600 \mathrm{~s}$ & $147^{\circ}$ \\
\hline & & NGC 4033 & $3600 \mathrm{~s}$ & $47^{\circ}$ \\
\hline & & NGC 4261 & $3600 \mathrm{~s}$ & $160^{\circ}$ \\
\hline & Feb. 18/19 & NGC 2663 & $3600 \mathrm{~s}$ & $98^{\circ}$ \\
\hline & & NGC 2872 & $3600 \mathrm{~s}$ & $32^{\circ}$ \\
\hline & & NGC 3260 & $3600 \mathrm{~s}$ & $0^{\circ}$ \\
\hline & & NGC 3557B & $3600 \mathrm{~s}$ & $107^{\circ}$ \\
\hline & & NGC 3557 & $3600 \mathrm{~s}$ & $30^{\circ}$ \\
\hline & & NGC 4697 & $3600 \mathrm{~s}$ & $160^{\circ}$ \\
\hline & Feb. 19/20 & UGC 4508 & $5400 \mathrm{~s}$ & $90^{\circ}$ \\
\hline & & NGC 3636 & $3600 \mathrm{~s}$ & $90^{\circ}$ \\
\hline & & UGC 7354 & $5400 \mathrm{~s}$ & $19^{\circ}$ \\
\hline & & NGC 5237 & $3600 \mathrm{~s}$ & $128^{\circ}$ \\
\hline
\end{tabular}


Table 3. Observation parameters

\begin{tabular}{|c|c|c|c|c|c|c|c|}
\hline date & $\begin{array}{c}\text { CCD } \\
\text { ESO-number }\end{array}$ & $\begin{array}{c}\text { spatial } \\
\text { resolution } \\
{[\operatorname{arcsec} / \text { pixel }]}\end{array}$ & ESO-number & $\begin{array}{c}\text { wavelength } \\
\text { range } \\
{[\AA]}\end{array}$ & $\begin{array}{c}\text { slit } \\
\text { width } \\
{[\operatorname{arcsec}]}\end{array}$ & $\begin{array}{c}\text { spectral } \\
\text { resolution } \\
{[\AA / \text { pixel }]}\end{array}$ & $\begin{array}{c}\text { instrumental } \\
\text { dispersion } \\
{\left[\mathrm{km} \mathrm{s}^{-1}\right]}\end{array}$ \\
\hline 1991 Mar. $24-25$ & 13 & 0.81 & 25 & $\overline{4253-6836}$ & 2.0 & 2.788 & 169 \\
\hline 1991 Mar. $25-28$ & 13 & 0.81 & 10 & $4835-5735$ & 1.7 & 0.974 & 51 \\
\hline 1992 Jan. 29 - Feb. 3 & 27 & 0.81 & 10 & $5162-6659$ & 2.0 & 0.970 & 53 \\
\hline 1992 Aug. 25 - 29 & 27 & 0.81 & 10 & $5175-6687$ & 2.0 & 0.969 & 39 \\
\hline 1993 Feb. $16-20$ & 24 & 0.81 & 10 & $5175-6687$ & 2.0 & 0.969 & 39 \\
\hline
\end{tabular}

Table 4. Measured emission lines of the sample galaxies

\begin{tabular}{|c|c|c|c|c|c|c|}
\hline galaxy & element & $\begin{array}{l}\lambda_{0} \\
{[\AA]} \\
\end{array}$ & $\begin{array}{c}\lambda \\
{[\AA]} \\
{[\AA]}\end{array}$ & $\begin{array}{c}F W H M \\
{[\AA]}\end{array}$ & $\begin{array}{c}v \\
{\left[\mathrm{~km} \mathrm{~s}^{-1}\right]}\end{array}$ & $\begin{array}{c}\sigma \\
{\left[\mathrm{km} \mathrm{s}^{-1}\right]}\end{array}$ \\
\hline \multirow[t]{6}{*}{ UGC 4508} & Fe II & 5591.38 & $\overline{56626.0}$ & 2.0 & 1856.2 & 44 \\
\hline & Fe I & 5595.06 & 5630.1 & 2.3 & 1877.5 & 50 \\
\hline & He I & 5875.6 & 5912.1 & 2.1 & 1862.4 & 43 \\
\hline & {$[\mathrm{N}$ iI $]$} & 6548.1 & 6588.7 & 2.3 & 1858.8 & 43 \\
\hline & $\mathrm{H} \alpha$ & 6562.8 & 6603.4 & $(4.6)$ & 1854.6 & $(85)$ \\
\hline & {$[\mathrm{N}$ II] } & 6583.4 & 6624.2 & 2.4 & 1859.3 & 44 \\
\hline \multirow[t]{3}{*}{ NGC 1889} & {$[\mathrm{~N}$ II $]$} & 6548.1 & 6603.2 & 5.0 & 2522.6 & 96 \\
\hline & $\mathrm{H} \alpha$ & 6562.8 & 6617.62 & 3.0 & 2504.2 & 56 \\
\hline & {$[\mathrm{N}$ II $]$} & 6583.4 & 6638.7 & 5.0 & 2518.2 & 95 \\
\hline \multirow[t]{8}{*}{ NGC 3125} & Fe II & 5591.38 & 5611.9 & 2.2 & 1100.2 & 48 \\
\hline & $\mathrm{Fe} \mathrm{I}$ & 5595.06 & 5615.8 & 2.2 & 1111.3 & 48 \\
\hline & He I & 5875.6 & 5896.73 & 2.5 & 1078.1 & 52 \\
\hline & Fe II & 6149.238 & 6171.83 & 2.6 & 1101.4 & 52 \\
\hline & {$[\mathrm{N}$ II $]$} & 6504.9 & 6528.552 & 2.7 & 1090.1 & 51 \\
\hline & {$[\mathrm{N}$ II $]$} & 6548.1 & 6571.7 & 2.6 & 1080.5 & 48 \\
\hline & $\mathrm{H} \alpha$ & 6562.8 & 6586.44 & 2.73 & 1079.9 & 50.7 \\
\hline & {$[\mathrm{N}$ iI $]$} & 6583.4 & 6607.26 & 2.6 & 1086.5 & 48 \\
\hline \multirow[t]{3}{*}{ NGC 5237} & {$[\mathrm{~N}$ II $]$} & 6548.1 & 6555.2 & $(6.1)$ & 325.1 & (114) \\
\hline & $\mathrm{H} \alpha$ & 6562.8 & 6570.07 & 2.3 & 332.1 & 42 \\
\hline & {$[\mathrm{N}$ iI $]$} & 6583.4 & 6590.7 & 2.4 & 332.4 & 45 \\
\hline \multirow[t]{10}{*}{ UGC 7354} & Fe II & 5619.9 & 5591.38 & 2.0 & 1529.2 & 44 \\
\hline & Fe I & 5623.9 & 5595.06 & 2.2 & 1545.3 & 48 \\
\hline & $\mathrm{Fe} \mathrm{I}$ & 5832.69 & 5804.06 & 1.9 & 1478.8 & 40 \\
\hline & Fe III & 5862.67 & 5833.65 & 2.8 & 1491.3 & 59 \\
\hline & Fe II & 6180.79 & 6149.238 & 2.2 & 1538.2 & 44 \\
\hline & {$[\mathrm{O}$ I] } & 6332.27 & 6300.3 & 2.1 & 1521.3 & 41 \\
\hline & {$[\mathrm{N}$ II $]$} & 6538.16 & 6504.9 & 2.4 & 1532.9 & 45 \\
\hline & {$[\mathrm{N}$ II $]$} & 6581.43 & 6548.1 & 2.2 & 1526.0 & 41 \\
\hline & $\mathrm{H} \alpha$ & 6595.7 & 6562.8 & $(5.3)$ & 1502.9 & (99) \\
\hline & {$[\mathrm{N}$ III } & 6616.878 & 6583.4 & 2.47 & 1524.5 & 45.9 \\
\hline
\end{tabular}

NGC 3904: Rotation disk $\left(r<6^{\prime \prime}\right)$ within the center, $h_{3} \propto-v$, indicating decoupled central component kinematics.

NGC 4105, NGC $4106=$ AM 1204-292: Gravitational interaction, reciprocal influence of kinematics. NGC 4105 features decoupled fast rotation within $2^{\prime \prime}$ of the galaxy's center, supported by the fact that $h_{3}$ resambles $-v$; NGC 4106 has an asymmetric rotational velocity profile and $h_{3}>0$. The latter is classified as lenticular in the ESO-Uppsala Sky Survey with a diffuse, round arm clearly visible, possibly produced by the interaction. RC3 classifies it for this reason as $\mathrm{SBb}$, however the color is more consistent with an S0 (Reduzzi \& Rampazzo 1996).
NGC 4697: Large E6 galaxy with only slight rotation, it also features a dust lane of intrinsically circular shape, located in the equitorial plane. The galaxy is flattened and may be an S0, the spheroidal part of NGC 4697 may well be a large bulge (Dejonghe et al. 1996).

NGC $\mathbf{5 2 3 7}=$ AM 1334-423: Nearby irregular galaxy, high-intensity emission lines, low continuum. This galaxy is believed to be a remnant of a spiral which collided with Centaurus A (NGC 5128) $510^{8}$ yr ago, lost half its disk material, which now forms the conspicuous ring of gas and dust line in Centaurus A, and was ejected as a non-rotating shred of dusty, gas-rich disc material which appears as a blue irregular/starburst dwarf galaxy 
(Thomson 1992).

NGC 6861 = IC 4949: Unusually high velocity dispersion at the center, fast rotation. This is a massive S0 galaxy, possibly influenced by a bar component. This galaxy also features a compact radio source in its core (Slee et al. 1994).

UGC $\mathbf{7 3 5 4}=\mathrm{A}$ 1216+04: Only emission lines, no absorption lines detected. This is a blue compact dwarf (Papaderos et al. 1996) or a starburst galaxy (Campos-Aguilar \& Moles 1991).

The ESO-Uppsala Sky Survey designates 25 galaxies as cluster members, 8 galaxies as group members, 3 galaxies as pairs (NGC 4105 with NGC 4106, NGC 5237 with a compact object) and 6 galaxies as field galaxies (IC 4797, NGC 2380, NGC 2865, NGC 2887, NGC 3302, NGC 3585). For 12 galaxies no such data was available.

Table 5 contains the most important measured or derived spectroscopic quantities of the sample galaxies. These mean parameters describe the individual galaxies sufficiently well for the investigation of correlations between different kinematic and non-kinematic properties and LOSVDs. All mean parameters refer to the galaxy properties within their effective radii.

- $v_{\text {sys }}$ is the heliocentric corrected system velocity;

- $v_{\text {rot }}$ is the rotational velocity, it was measured as the difference between an average of the radial velocities where the rotation curve flattened and the central velocity;

$-\mathrm{d} v / \mathrm{d} r_{0}$ is the gradient of the rotation curve at the center of the galaxy;

- $\sigma_{0}$ is the mean value of all velocity dispersions within $5 \operatorname{arcsec}^{5}$ of the galaxy's center;

- $\sigma_{\mathrm{m}}$ was derived by averaging all velocity dispersions within $r_{\mathrm{e} / 2}{ }^{6}$;

- $\sigma_{\mathrm{e} / 2}$ is the velocity dispersion at half of the effective radius:

- $\Delta \sigma_{\mathrm{e} / 2}$ is the relative change of the velocity dispersion and is calculated as $\Delta \sigma_{\mathrm{e} / 2}=\left(\sigma_{\mathrm{e} / 2}-\sigma_{0}\right) / \sigma_{0}$;

- $(v / \sigma)^{*}$ is the anisotropy parameter and is calculated

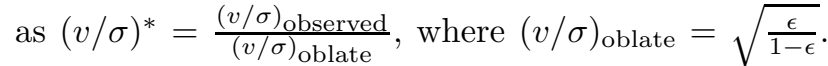
The ellipticities of the sample galaxies were taken from RC3.

\section{Correlations between kinematic and photometric parameters}

The photometric parameters used for these correlation were taken from Burstein et al. (1987), RC3 and the ESOUppsala Sky Survey, kinematic quantities are results of this work.

\footnotetext{
${ }^{5}$ Consistent with Faber et al. (1989).

${ }^{6}$ Consistent with Bender et al. (1994).
}

To calculate the distances of the sample galaxies two different models were used:

1. The distances were calculated by assuming an undisturbed Hubble-flow and $H_{0}=75 \mathrm{~km} \mathrm{~s}^{-1} \mathrm{Mpc}^{-1}$, using the system velocities taken from Faber et al. (1989).

2. The distances were taken from the catalogue of KraanKorteweg (1986), where a model of the Virgo cluster infall was added to the Hubble flow.

\subsection{Correlations between anisotropy and photometric parameters}

Binney (1978) was the first to utilize a $(v / \sigma)$ vs. $\epsilon$-diagram in order to get a statistic reference if a group of galaxies suits into the model of an oblate rotator or whether it rather consists of dynamical hot systems, where chaotic star motion dominates.

In Fig. $1(v / \sigma)^{*}$ vs. $\epsilon,(v / \sigma)$ vs. $\epsilon$ and $v$ vs. $\epsilon$ are plotted for all sample galaxies where these parameters are available. Galaxies with $(v / \sigma)^{*}>1$ are expected to be rotationally supported systems. These are (sorted by increasing $\epsilon$ ): NGC 1404, NGC 2872, NGC 3260, NGC 4105, NGC 7196, NGC 3557, NGC 3268, NGC 6861, NGC 2271, NGC 7049 and NGC 1537. The last four objects are S0 systems, the others are elliptical galaxies with fast rotation.

The correlation between anisotropy and luminosity was first described in Davies et al. (1983). Galaxies with low luminosity are mostly rotationally supported systems while galaxies with high luminosity are generally systems with high anisotropy. The existence of this correlation is explained by the increasing influence of chaotic relaxation and the increasing influence of merging with increasing galaxy mass (and therefore luminosity) (Davies et al. 1983; Bender et al. 1992). In Fig. 2 the correlation between $(v / \sigma)^{*}$ and $M_{B}$ is plotted.

The galaxy NGC 3557 is represented by the data point with the greatest luminosity and doesn't follow the general trend. This E3-galaxy features high luminosity and a rotational velocity of $v_{\text {rot }}=217 \pm 11 \mathrm{~km} \mathrm{~s}^{-1}$.

\section{2. $\kappa$-space}

$\kappa$-space was introduced by Bender et al. (1992) and was originally defined to examine the physical properties of dynamically hot galaxies. The axes of $\kappa$-space are proportional to the logarithm of galaxy mass $\left(\kappa_{1}\right)$, mass-to-light ratio $\left(\kappa_{3}\right)$, and a third quantity that is mainly surface brightness $\left(\kappa_{2}\right)$ (Burstein et al. 1997). The $\kappa_{1} / \kappa_{3}$-projection represents a side-view of the fundamental plane, the $\kappa_{1} / \kappa_{2}$-projection shows it nearly face-on.

All sample galaxies where the necessary input parameters $\sigma_{\mathrm{c}}, R_{\mathrm{e}}$ and $I_{\mathrm{e}}$ were available are represented in Fig. 3. 
Table 5. Measured spectroscopic parameters of the sample galaxies. Quantities marked with ${ }^{*}$ are results of emission line measurements

\begin{tabular}{|c|c|c|c|c|c|c|c|c|}
\hline galaxy & $\begin{array}{c}v_{\text {sys }} \\
{\left[\mathrm{km} \mathrm{s}^{-1}\right]}\end{array}$ & $\begin{array}{c}v_{\text {rot }} \\
{\left[\mathrm{km} \mathrm{s}^{-1}\right]}\end{array}$ & $\begin{array}{c}\mathrm{d} v / \mathrm{d} r_{0} \\
{\left[\mathrm{~km} \mathrm{~s}^{-1} \operatorname{arcsec}^{-1}\right]}\end{array}$ & $\begin{array}{c}\sigma_{0} \\
{\left[\mathrm{~km} \mathrm{~s}^{-1}\right]}\end{array}$ & $\begin{array}{c}\sigma_{\mathrm{m}} \\
{\left[\mathrm{km} \mathrm{s}^{-1}\right]}\end{array}$ & $\begin{array}{c}\sigma_{\mathrm{e} / 2} \\
{\left[\mathrm{~km} \mathrm{~s}^{-1}\right]}\end{array}$ & $\Delta \sigma_{\mathrm{e} / 2}$ & $(v / \sigma)^{*}$ \\
\hline I1729 & $1441 \pm 3$ & $54 \pm 4$ & $21 \pm 8$ & $131 \pm 7$ & $130 \pm 9$ & $127 \pm 18$ & $-0.03 \pm 0.14$ & $0.45 \pm 0.05$ \\
\hline N1404 & $1834 \pm 4$ & $113 \pm 10$ & $1 \pm 9$ & $259 \pm 5$ & $247 \pm 4$ & $216 \pm 10$ & $-0.17 \pm 0.04$ & $1.30 \pm 0.11$ \\
\hline N1427 & $1417 \pm 3$ & $63 \pm 10$ & $9 \pm 5$ & $157 \pm 4$ & $150 \pm 8$ & $150 \pm 8$ & $-0.05 \pm 0.06$ & $0.61 \pm 0.10$ \\
\hline N1537 & $1407 \pm 2$ & $176 \pm 8$ & $18 \pm 4$ & $138 \pm 4$ & $121 \pm 4$ & $102 \pm 11$ & $-0.26 \pm 0.08$ & $2.03 \pm 0.11$ \\
\hline N1549 & $1198 \pm 2$ & $53 \pm 12$ & $0 \pm 3$ & $218 \pm 3$ & $191 \pm 4$ & $109 \pm 11$ & $-0.50 \pm 0.05$ & $0.61 \pm 0.14$ \\
\hline N1889 & $2406 \pm 6$ & $113 \pm 13$ & $9 \pm 18$ & $167 \pm 11$ & - & - & - & - \\
\hline & $2524 \pm 8^{*}$ & - & - & $89.4 \pm 19.5^{*}$ & - & - & - & - \\
\hline N2271 & $2558 \pm 6$ & $263 \pm 36$ & $20 \pm 7$ & $237 \pm 7$ & $228 \pm 8$ & $235 \pm 8$ & $-0.01 \pm 0.04$ & $1.72 \pm 0.24$ \\
\hline N2325 & $2073 \pm 5$ & $9 \pm 17$ & $5 \pm 17$ & $206 \pm 8$ & $215 \pm 13$ & $179 \pm 27$ & $-0.13 \pm 0.13$ & $0.05 \pm 0.09$ \\
\hline N2380 & $1755 \pm 4$ & $68 \pm 16$ & $4 \pm 10$ & $200 \pm 6$ & - & - & - & - \\
\hline N2434 & $1358 \pm 4$ & $41 \pm 15$ & $7 \pm 10$ & $189 \pm 6$ & $170 \pm 8$ & $152 \pm 10$ & $-0.20 \pm 0.06$ & $0.89 \pm 0.33$ \\
\hline $\mathrm{I} 2311$ & $1636 \pm 4$ & $30 \pm 18$ & $7 \pm 8$ & $211 \pm 5$ & $189 \pm 5$ & $143 \pm 13$ & $-0.32 \pm 0.07$ & $0.50 \pm 0.30$ \\
\hline U4508 & $1865 \pm 8^{*}$ & - & - & $44.9 \pm 3.1^{*}$ & - & - & - & - \\
\hline N2663 (major axis) & $1987 \pm 8$ & $35 \pm 31$ & $6 \pm 19$ & $329 \pm 11$ & - & - & - & - \\
\hline $\mathrm{N} 2663\left(\mathrm{PA}=98^{\circ}\right)$ & $1951 \pm 8$ & $13 \pm 19$ & $3 \pm 13$ & $321 \pm 9$ & - & - & - & - \\
\hline N2699 & $1715 \pm 3$ & $73 \pm 6$ & $17 \pm 5$ & $152 \pm 6$ & - & - & - & - \\
\hline N2887 & $2843 \pm 6$ & $6 \pm 20$ & $2 \pm 19$ & $296 \pm 8$ & $267 \pm 7$ & $187 \pm 9$ & $-0.37 \pm 0.04$ & $0.03 \pm 0.12$ \\
\hline N2865 & $2529 \pm 4$ & $72 \pm 15$ & $1 \pm 10$ & $184 \pm 5$ & $182 \pm 5$ & $175 \pm 8$ & $-0.05 \pm 0.05$ & $0.67 \pm 0.14$ \\
\hline N2872 & $3117 \pm 9$ & $190 \pm 50$ & $8 \pm 16$ & $281 \pm 13$ & $248 \pm 10$ & $154 \pm 29$ & $-0.45 \pm 0.11$ & $1.98 \pm 0.53$ \\
\hline N2888 & $2342 \pm 3$ & $54 \pm 9$ & $14 \pm 6$ & $138 \pm 7$ & $140 \pm 7$ & $141 \pm 9$ & $0.02 \pm 0.08$ & $0.65 \pm 0.11$ \\
\hline N2986 & $2174 \pm 7$ & $57 \pm 12$ & $30 \pm 10$ & $282 \pm 13$ & $243 \pm 14$ & $206 \pm 31$ & $-0.27 \pm 0.11$ & $0.61 \pm 0.13$ \\
\hline N3078 & $2428 \pm 5$ & $99 \pm 8$ & $30 \pm 18$ & $305 \pm 6$ & $292 \pm 9$ & $255 \pm 16$ & $-0.16 \pm 0.05$ & $0.75 \pm 0.06$ \\
\hline N3087 & $2511 \pm 4$ & $34 \pm 8$ & $13 \pm 5$ & $259 \pm 6$ & $246 \pm 7$ & $192 \pm 24$ & $-0.26 \pm 0.10$ & $0.30 \pm 0.07$ \\
\hline N3136 & $1670 \pm 5$ & $10 \pm 16$ & $2 \pm 16$ & $226 \pm 5$ & $197 \pm 5$ & $115 \pm 12$ & $-0.49 \pm 0.06$ & $0.07 \pm 0.12$ \\
\hline N3125 & $1077 \pm 11^{*}$ & - & - & $51.9 \pm 4.0^{*}$ & - & - & - & - \\
\hline N3224 & $2898 \pm 8$ & $94 \pm 26$ & $25 \pm 10$ & $216 \pm 16$ & $206 \pm 13$ & $172 \pm 19$ & $-0.20 \pm 0.11$ & $0.94 \pm 0.26$ \\
\hline N3250 & $2704 \pm 5$ & $132 \pm 9$ & $21 \pm 10$ & $298 \pm 6$ & $262 \pm 6$ & $188 \pm 16$ & $-0.37 \pm 0.06$ & $0.81 \pm 0.06$ \\
\hline N3258 & $2646 \pm 5$ & $39 \pm 9$ & $14 \pm 14$ & $357 \pm 6$ & $319 \pm 6$ & $267 \pm 18$ & $-0.25 \pm 0.05$ & $0.29 \pm 0.07$ \\
\hline N3260 & $2248 \pm 6$ & $211 \pm 16$ & $39 \pm 12$ & $174 \pm 9$ & $147 \pm 10$ & $106 \pm 24$ & $-0.39 \pm 0.14$ & $2.71 \pm 0.27$ \\
\hline N3268 & $2575 \pm 6$ & $211 \pm 38$ & $47 \pm 21$ & $197 \pm 10$ & $177 \pm 10$ & $122 \pm 17$ & $-0.38 \pm 0.09$ & $1.91 \pm 0.36$ \\
\hline N3302 & $3742 \pm 10$ & $18 \pm 41$ & $12 \pm 15$ & $379 \pm 17$ & $309 \pm 21$ & $148 \pm 21$ & $-0.61 \pm 0.06$ & $0.10 \pm 0.22$ \\
\hline N3309 & $4024 \pm 6$ & $17 \pm 10$ & $12 \pm 9$ & $282 \pm 10$ & - & - & - & - \\
\hline N3377 & $591 \pm 2$ & $86 \pm 4$ & $25 \pm 7$ & $135 \pm 7$ & $115 \pm 8$ & $81 \pm 22$ & $-0.40 \pm 0.17$ & $0.88 \pm 0.07$ \\
\hline N3557B & $2723 \pm 4$ & $124 \pm 7$ & $32 \pm 11$ & $201 \pm 6$ & - & - & - & - \\
\hline N3557 & $2885 \pm 7$ & $217 \pm 11$ & $26 \pm 7$ & $363 \pm 14$ & $276 \pm 7$ & $181 \pm 17$ & $-0.50 \pm 0.05$ & $1.26 \pm 0.07$ \\
\hline N3585 & $1333 \pm 2$ & $157 \pm 3$ & $19 \pm 4$ & $232 \pm 3$ & $218 \pm 3$ & $220 \pm 8$ & $-0.06 \pm 0.04$ & $0.80 \pm 0.02$ \\
\hline N3617 & $1955 \pm 3$ & $99 \pm 20$ & $9 \pm 4$ & $111 \pm 8$ & $111 \pm 7$ & $109 \pm 20$ & $-0.02 \pm 0.20$ & $1.40 \pm 0.30$ \\
\hline N3636 & $1609 \pm 4$ & $8 \pm 14$ & $4 \pm 6$ & $114 \pm 10$ & - & - & - & - \\
\hline N3904 & $1592 \pm 3$ & $69 \pm 7$ & $18 \pm 5$ & $211 \pm 4$ & $195 \pm 5$ & $118 \pm 14$ & $-0.44 \pm 0.07$ & $0.57 \pm 0.06$ \\
\hline N3923 & $1639 \pm 4$ & $53 \pm 13$ & $4 \pm 10$ & $290 \pm 4$ & $275 \pm 6$ & $176 \pm 22$ & $-0.39 \pm 0.08$ & $0.27 \pm 0.07$ \\
\hline N4033 & $1452 \pm 3$ & $99 \pm 8$ & $10 \pm 6$ & $134 \pm 4$ & $142 \pm 4$ & $161 \pm 12$ & $0.20 \pm 0.10$ & $0.60 \pm 0.05$ \\
\hline N4105 & $2147 \pm 3$ & $158 \pm 14$ & $19 \pm 8$ & $181 \pm 4$ & $165 \pm 4$ & $133 \pm 6$ & $-0.27 \pm 0.04$ & $1.62 \pm 0.15$ \\
\hline N4106 & $1861 \pm 4$ & $10 \pm 5$ & $9 \pm 11$ & $230 \pm 5$ & $216 \pm 6$ & $169 \pm 9$ & $-0.27 \pm 0.04$ & $0.09 \pm 0.05$ \\
\hline U7354 & $1505 \pm 18^{*}$ & - & - & $46.1 \pm 4.9^{*}$ & - & - & - & - \\
\hline N4261 & $2038 \pm 5$ & $12 \pm 16$ & $1 \pm 13$ & $356 \pm 5$ & $294 \pm 4$ & $224 \pm 14$ & $-0.37 \pm 0.04$ & $0.12 \pm 0.16$ \\
\hline $\mathrm{N} 4697\left(\mathrm{PA}=100^{\circ}\right)$ & $1050 \pm 3$ & $97 \pm 7$ & $27 \pm 4$ & $187 \pm 6$ & $172 \pm 9$ & $164 \pm 12$ & $-0.12 \pm 0.07$ & $0.77 \pm 0.07$ \\
\hline N4697 (minor axis) & $1088 \pm 3$ & $27 \pm 10$ & $6 \pm 4$ & $181 \pm 4$ & $174 \pm 4$ & $177 \pm 11$ & $-0.02 \pm 0.06$ & $0.21 \pm 0.08$ \\
\hline N5061 & $1931 \pm 3$ & $59 \pm 13$ & $2 \pm 7$ & $220 \pm 4$ & $213 \pm 4$ & $229 \pm 13$ & $0.04 \pm 0.06$ & $0.66 \pm 0.15$ \\
\hline N5237 & $302 \pm 3^{*}$ & - & - & $48.5 \pm 1.0^{*}$ & - & - & - & - \\
\hline N5903 & $2528 \pm 4$ & $9 \pm 17$ & $4 \pm 11$ & $212 \pm 6$ & $194 \pm 6$ & $177 \pm 16$ & $-0.16 \pm 0.08$ & $0.08 \pm 0.17$ \\
\hline I4497 & $2635 \pm 3$ & $137 \pm 6$ & $28 \pm 9$ & $245 \pm 5$ & $219 \pm 4$ & $159 \pm 12$ & $-0.35 \pm 0.05$ & $0.56 \pm 0.03$ \\
\hline N6861 & $2752 \pm 7$ & $240 \pm 10$ & $45 \pm 11$ & $382 \pm 7$ & $340 \pm 6$ & $297 \pm 19$ & $-0.22 \pm 0.05$ & $0.96 \pm 0.04$ \\
\hline N7029 & $2703 \pm 5$ & $132 \pm 7$ & $29 \pm 6$ & $201 \pm 7$ & $177 \pm 6$ & $106 \pm 12$ & $-0.48 \pm 0.06$ & $0.82 \pm 0.05$ \\
\hline N7049 & $2183 \pm 5$ & $171 \pm 17$ & $20 \pm 12$ & $267 \pm 7$ & $220 \pm 7$ & $114 \pm 23$ & $-0.57 \pm 0.09$ & $1.16 \pm 0.12$ \\
\hline N7196 & $2800 \pm 7$ & $243 \pm 25$ & $40 \pm 18$ & $260 \pm 12$ & $234 \pm 9$ & $229 \pm 24$ & $-0.12 \pm 0.10$ & $1.76 \pm 0.19$ \\
\hline I5297 & $2831 \pm 6$ & $113 \pm 25$ & $5 \pm 19$ & $267 \pm 7$ & - & - & - & - \\
\hline
\end{tabular}




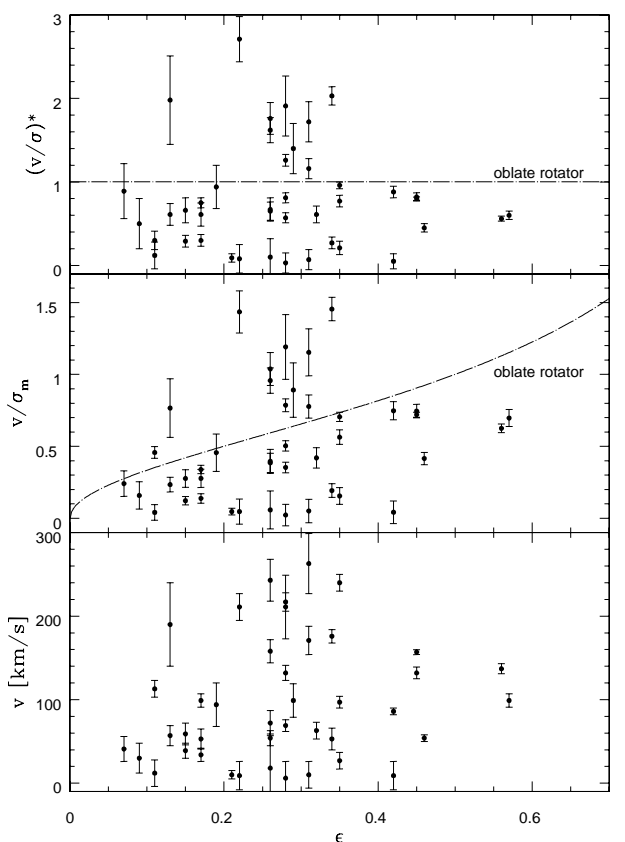

Fig. 1. $(v / \sigma)^{*}$ vs. $\epsilon,(v / \sigma)$ vs. $\epsilon$ and $v$ vs. $\epsilon$ - diagrams. The dotted line represents the theoretical model of an oblate rotator

The data point of one galaxy, NGC 6861, lies within the "zone of exclusion" (ZOE) $\kappa_{1}+\kappa_{2}>8.0$ defined by Burstein et al. (1997). All galaxies are giant sequence (Gas-Stellar Continuum) members. In the giant sequence $\kappa_{2}$ increases while $\kappa_{1}$ decreases, it contains bulges, normal ellipticals (giant and intermediate) and compacts.

\subsection{Statistics}

Rotational velocity profiles, velocity dispersion profiles, $h_{3}$ and $h_{4}$-profiles were extracted for 49 galaxies. Bender et al. (1994) identified several kinematic types by their rotation, velocity dispersion, $h_{3}$ and $h_{4}$ profiles, finding correlations between kinematic profiles and photometric properties like diskyness or boxyness. Following this description, our sample galaxies were divided into the kinematic types, the kinematic types of the individual galaxies are listed in Table 6 .

$$
\begin{aligned}
& \text { galaxies with decoupled } \\
& \text { central component kinematics } 17 \text { galaxies }=32 \% \\
& \text { disky } \ldots \ldots \ldots \ldots \ldots \ldots \ldots . \ldots \text { galaxies }=13 \% \\
& \text { boxy, without rotation ...... } 10 \text { galaxies }=19 \% \\
& \text { boxy, with weak rotation ... } 3 \text { galaxies }=6 \% \\
& \text { E0 .................... } 4 \text { galaxies }=8 \% \\
& \text { normal E-galaxies ......... } 7 \text { galaxies }=13 \% \\
& \text { unknown } \ldots \ldots \ldots \ldots \ldots \ldots . \quad 3 \text { galaxies }=6 \% \\
& \text { not classifiable } \ldots \ldots \ldots \ldots \ldots .9 \text { galaxies }=17 \% \text {. }
\end{aligned}
$$

Galaxies where not enough data for this evaluation

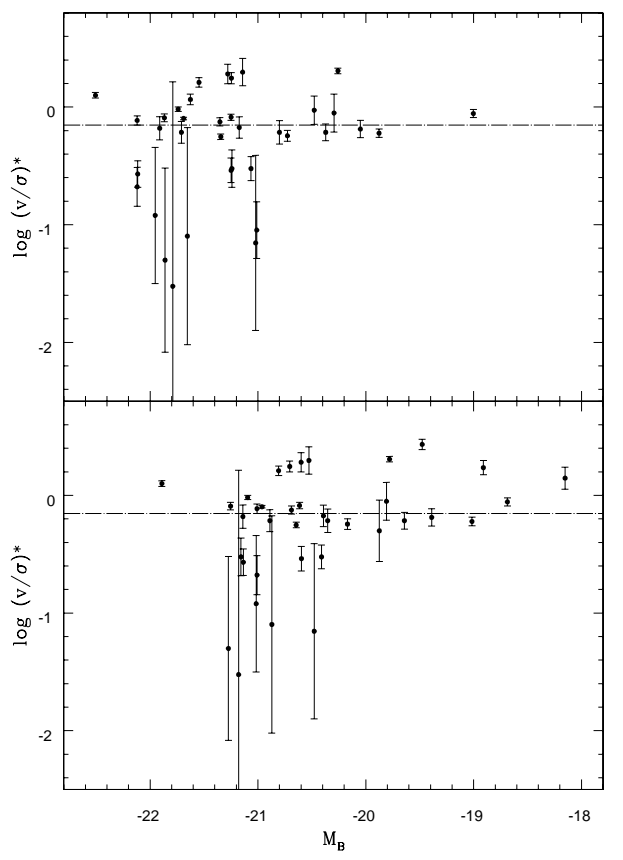

Fig. 2. The correlation between anisotropy and absolute luminosity in the $B$ filter. The distances of the galaxies needed for the calculation of $M_{B}$ were in the upper diagram taken from the catalog of Kraan-Korteweg (1986) and in the lower diagram derived under the assumption of an undisturbed Hubble flow. The dotted line represents the dividing line between rotational supported systems and anisotropic galaxies $(v / \sigma)^{*}=0.7$ given by Bender et al. (1992)

was obtainable are classified as "unknown", galaxies with profiles which did not fit into any of the representative object classes are found in "not classifiable". Some galaxies fit into more than one class, they are multiply counted.

Disky early-type galaxies are two-component systems with a hot bulge and a cold, fast rotating disk. This superposition will cause asymmetric LOSVDs which are characterized by strong negative $h_{3}$ values. Features in the major axis rotation curve that are caused by varying light contributions of the disk are reflected by similar features in the $h_{3}$ profile. Boxy ellipticals show none or only weak rotation and positive $h_{4}$ values on their major axes. Weak rotation causes some weak asymmetry in their LOSVDs and therefore slightly negative $h_{3}$ values. E0 galaxies do show a slight increase of velocity dispersion within their photometric core radii and smaller $h_{4}$ values inside their core radii than further out. Normal E-galaxies are galaxies with $h_{3}$ and $h_{4} \approx 0$.

$32 \%$ of all galaxies contain kinematically decoupled central components. The size of these central components was found to be $0.40 \pm 0.28 \mathrm{kpc}$ and in all cases less than $1 \mathrm{kpc}$. The identification of decoupled central components is based on the kinematic profiles, which show features typical for disky galaxies, but $h_{3}$ has the opposite sign 
Table 6. Kinematic types of the sample galaxies following Bender et al. (1994)

\begin{tabular}{clcl}
\hline galaxy & kinematic type & galaxy & kinematic type \\
\hline \hline I1729 & disky: & N3302 & boxy, without rotation: \\
N1404 & decoupled central component & N3309 & normal E-galaxy \\
N1427 & decoupled central component & N3377 & normal E-galaxy \\
N1537 & normal E-galaxy & N3557B & decoupled central component \\
N1549 & E0; boxy, with weak rotation & N3557 & normal E-galaxy \\
N1889 & unknown & N3585 & decoupled central component; disky \\
N2271 & decoupled central component; disky & N3617 & decoupled central component: \\
N2325 & not classifiable & N3260 & disky \\
N2434 & boxy, without rotation & N3636 & E0; boxy, without rotation \\
N2380 & boxy, without rotation & N3904 & decoupled central component \\
I2311 & decoupled central component; disky & N3923 & not classifiable \\
U4508 & not classifiable & N4033 & decoupled central component \\
N2663 & boxy, without rotation & N4105 & decoupled central component \\
N2699 & decoupled central component & N4106 & unknown \\
N2887 & boxy, without rotation & U7354 & not classifiable \\
N2865 & boxy, without rotation: & N4261 & boxy, without rotation \\
N2872 & unknown & N4697 & normal E-galaxy \\
N2888 & decoupled central component & N5061 & E0; boxy, with weak rotation \\
N2986 & decoupled central component & N5237 & not classifiable \\
N3078 & decoupled central component & N5903 & boxy, without rotation \\
N3087 & decoupled central component & I4797 & disky \\
N3136 & not classifiable & N6861 & not classifiable \\
N3125 & not classifiable & N7029 & not classifiable \\
N3224 & normal E-galaxy: & N7049 & normal E-galaxy \\
N3250 & decoupled central component: & N7196 & decoupled central component: \\
N3258 & boxy, with weak rotation & I5297 & boxy, without rotation \\
N3268 & disky & & \\
\hline
\end{tabular}

to $v$ and the reversal in the rotation corresponds to the radius where also $h_{3}$ changes sign. On comparing these galaxies with results in literature, a good accordance was found, e.g. NGC 3078 and NGC 3250 where central depressions in the $\mathrm{Mg}_{2}$ profiles were identified by Carollo et al. (1993). For two of the sample galaxies central surfacebrightness profiles have been obtained by HST, NGC 3377 and NGC 4697 (Faber et al. 1997). Both have power-law profiles which lack cores. NGC 4261 and NGC 4697 do contain nuclear dust structures also indentified by HST (van Dokkum \& Franx 1995).

The percentage of sample galaxies where a disk component was evident is altogether $49 \%$, in $36 \%$ no disk component could be detected and in $15 \%$ of the sample galaxies this is uncertain. It may therefore be concluded that more than half of the sample galaxies contain stellar disk components, adding the fact that with greater spatial resolution even smaller disks might have been detected.

The luminosity distributions of individual kinematic object classes are shown in Fig. 4. Galaxies with kinematically decoupled components are evenly distributed in the luminosity range, in contrary to Bender (1996) who find that these components should be more frequent in luminous ellipticals. The dichotomy in the class of elliptical galaxies in boxy and disky objects is confirmed also from the kinematic point of view. However it is noteworthy that some disky ellipticals show the signature of a kinematically decoupled central component.

It is an accepted fact that merging plays an important role in the evolution of early-type galaxies up to altering the original morphological type (Barnes 1996). The most notable signature of such an event are kinematically decoupled gaseous and stellar components (Bertola et al. 1990; Bender 1996). Statistics of our sample galaxies were used in order to verify this picture: ZCAT (Huchra et al. 1995) was searched for galaxies in the vicinity of each sample member using radial bins of 50, 100, 150 and $200 \mathrm{kpc}$ around each galaxy and a redshift interval of $\pm 1000 \mathrm{~km} \mathrm{~s}^{-1}$. The resulting histogram (Fig. 5) reflects the well-known fact that generally elliptical galaxies are located in high-density environments (Dressler et al. 1994). However, it is noteworthy that the subsample of objects with decoupled components are found in groups of even higher density than average.

The peculiar central component kinematics in some ellipticals can possibly also be explained in ways different from the above scenario. E.g. the decoupled component could be due to streaming in a triaxial body, obliquely projected (Binney 1985; Franx et al. 1991; Statler 1994); but rotation amplitudes in the central components are in general too high and central metallicities are enhanced with respect to the main body, so this scenario cannot 


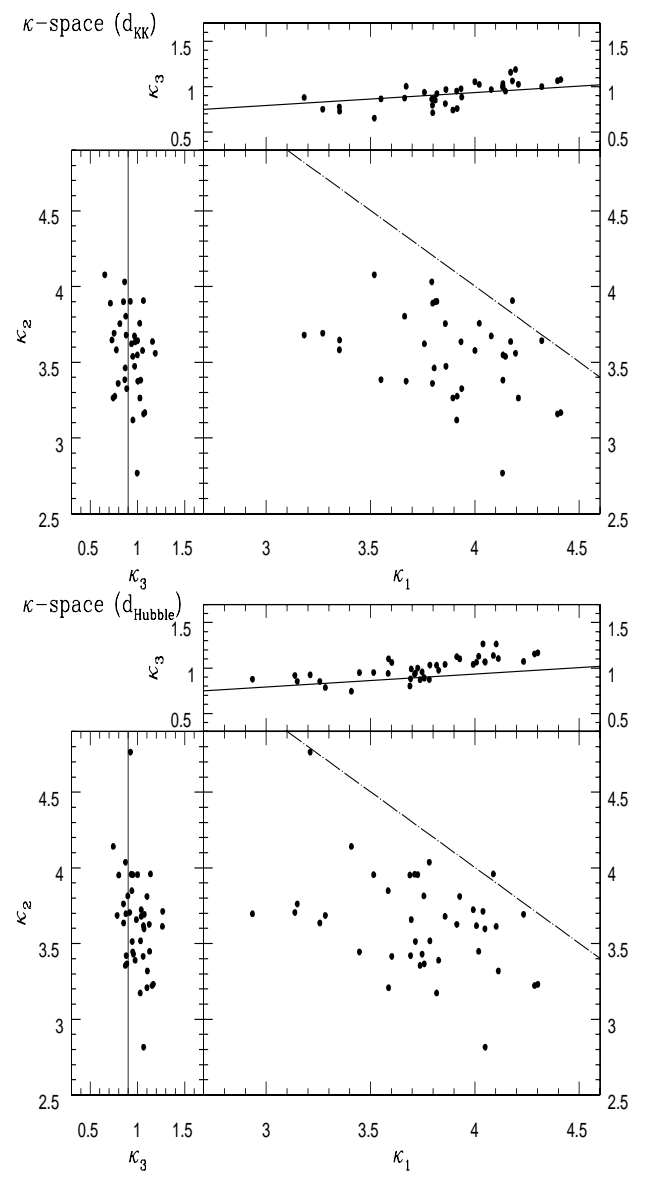

Fig. 3. $\kappa$-space in the parameterization published by Bender et al. (1992). The distances of the galaxies needed for the calculation of $R_{\mathrm{e}}$ were in the upper diagram taken from the cata$\log$ of Kraan-Korteweg (1986) and in the lower diagram derived under the assumption of an undisturbed Hubble flow. The dotted line in the $\kappa_{1} / \kappa_{2}$-plot marks the beginning of the "zone of exclusion" (ZOE), which extends to the upper right. The full line in the other projections marks the fundamental plane

account for the formation of the majority of ellipticals with peculiar central components (Bender 1996).

\section{Conclusions}

We have measured the line-of-sight velocity distributions (LOSVDs) for a sample of 49 early-type galaxies by means of the fourier quotient method and the fourier fitting method. From the observed LOSVDs rotation velocities and velocity dispersions were derived and deviations of the LOSVDs from Gaussian profiles were parameterized by the amplitudes $h_{3}$ and $h_{4}$ of third- and fourth order Gauss-Hermite functions. For the investigation of correlations between different kinematic and photometric properties mean kinematic parameters were derived from the profiles.

The galaxies are shown to follow the general correlation between anisotropy and luminosity as described by

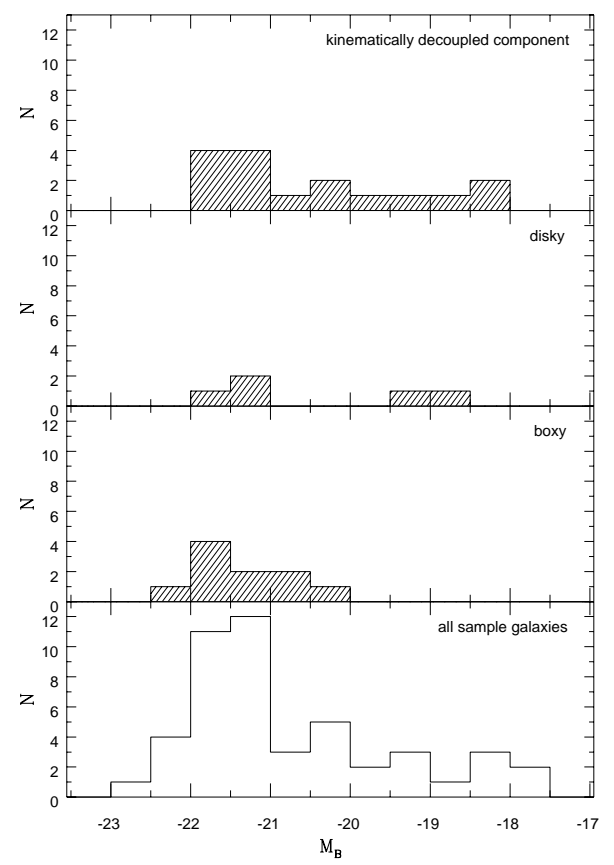

Fig. 4. Absolute luminosity distributions of the sample galaxies with respect to the kinematic types introduced by Bender et al. (1994). The shaded distributions represent respective luminosity distributions of the subsamples with kinematically decoupled component, boxiness or diskiness. Note that "diskiness" and "boxiness" were infered from the galaxy's kinematic profiles, not photometric properties

Davies et al. (1983). The position of the galaxies in $\kappa$ space (Bender et al. 1992) was also plotted. All galaxies are shown to be giant sequence members, one galaxy lies within the zone of exclusion (ZOE).

Following Bender et al. (1994) the sample galaxies were divided into several representative object classes by examining their kinematic profiles. $32 \%$ of the examined galaxies contain kinematically decoupled central components, the size of these components was $0.40 \pm 0.28 \mathrm{kpc}$, in each case the component was smaller than $1 \mathrm{kpc}$. The kinematic signature of a stellar disk component is found in $49 \%$ of the sample galaxies, in $15 \%$ this is uncertain. Therefore it is highly probable that in more than half of all early-type galaxies a stellar disk component exists.

The galaxies which contain kinematically decoupled components are more likely to be found in groups of higher density than others. This fact supports the theory that kinematically decoupled components are the remnants of merging events which are more likely to be found in highdensity environments than in the field.

\section{References}

Barnes J., 1996, "Interaction and Induced Star Formation",

Saas-Fee Advanced Course 26, Lecture Notes 1996, p. 275 Bender R., Surma P., 1992, A\&A 258, 250 


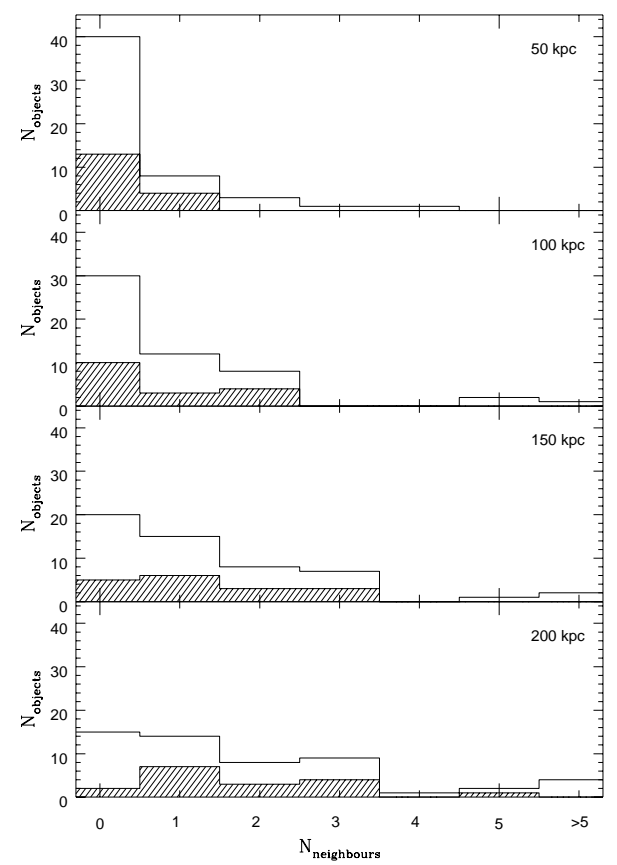

Fig. 5. Histograms of the number of sample galaxies with neighbours within a redshift interval of $\pm 1000 \mathrm{~km} \mathrm{~s}^{-1}$ using ZCAT. The histograms range from a radial distance between sample galaxies and neighbours from $0<r<50 \mathrm{kpc}$ (uppermost diagram) to $150<r<200 \mathrm{kpc}$ (lowest diagram). The histograms for sample galaxies with kinematically decoupled components are shown as shaded. The last $N_{\text {neighbour }}$ bin includes all galaxies with more than 5 nearby galaxies. A trend that galaxies with kinematically decoupled components are more likely to be located in crowded environments (where $N_{\text {neighbours }}$ is higher) is evident

Bender R., Burstein D., Faber S.M., 1992, ApJ 399, 462

Bender R., Saglia R.P., Gerhard O.E., 1994, MNRAS 269, 785

Bender R., 1996, IAU Symp. 171 "New Light on Galaxy Evolution", Bender R., Davies R.L. (eds.). Kluwer, Netherlands, p. 181

Bertola F., et al., 1990, "Dynamics and Interactions of Galaxies", Wielen (ed.), p. 249

Binney J.J., 1978, MNRAS 183, 501

Binney J.J., 1985, MNRAS 212, 767

Binney J.J., Davies R.L., Illingworth G.D., 1990, ApJ 361, 78

Burstein D., Davies R.L., Dressler A., et al., 1987, ApJS 64, 601

Burstein D., Bender R., Faber S.M., Nolthenius R., 1997, AJ 114, 1365

Campos-Aguilar A., Moles M., 1991, A\&A 241, 358

Carollo C.M., Danziger I.J., Buson L., 1993, MNRAS 265, 553

Carollo C.M., Danziger I.J., 1994a, MNRAS 270, 523

Carollo C.M., Danziger I.J., 1994b, MNRAS 270, 743

Danziger I.J., Goss W.M., 1983, MNRAS 202, 703

Davies R.L., et al., 1983, ApJ 266, 41

Dejonghe H., et al., 1996, A\&A 306, 363 de Vaucouleurs G., de Vaucouleurs A., Corwin H.G., et al., 1991, "Third Reference Catalogue of Bright Galaxies". Springer Verlag (RC3)

Dressler A., Oemler A., Butcher H.R., Gunn J.E., 1994, ApJ 430, 107

Faber S.M., Wegner G., Burstein D., et al., 1989, ApJS 69, 763

Faber S.M., Tremaine S., Ajhar E.A., et al., 1997, AJ 114, 1771

Franx M., Illingworth G., Heckman T., 1989, ApJ 344, 613

Franx M., Illingworth G., de Zeeuw T., 1991, ApJ 383, 112

Huchra J.P., Geller M.J., Corwin H.G., 1995, "The CfA Redshift Catalogue", Harvard-Smithsonian Center for Astrophysics (ZCAT)

Kraan-Korteweg R.C., 1986, A\&AS 66, 255

Lauberts A., Valentijn E.A., 1989, "The Surface Photometry Catalogue of the ESO-Uppsala Galaxies", ESO, ESOUppsala

Liu C.T., Kennicutt R.C., 1995, ApJ 450, 547

Marlowe A.T., Meurer G.R., Heckman T.M., Schommer R., 1997, ApJS 112, 285

Papaderos P., Loose H.-H., Thuan T.X., Fricke K.J., 1996, A\&AS 120, 207

Paturel G., Fouque P., Bottinelli L., Gouguenheim L., 1989, A\&AS 80, 299, "Catalogue of Principal Galaxies" (PGC)

Reduzzi L., Rampazzo R., 1996, A\&AS 116, 515

Sadler E.M., Jenkins C.R., Kotanyi C.G., 1989, MNRAS 240, 591

Sandage A., Tammann G.A., 1981, "Revised Shapley-Ames Catalog of Bright Galaxies". Carnegie Institution of Washington Publications, p. 635 (RSA)

Slee O.B., Sadler E.M., Reynolds J.E., Ekers R.D., 1994, MNRAS 269, 928

Statler T.S., 1994, ApJ 425, 500

Telles E., Melnick J., Terlevich R., 1997, MNRAS 288, 78

Thomson R.C., 1992, MNRAS 257, 689

van der Marel R.P., Franx M., 1993, ApJ 407, 525

van Dokkum P.G., Franx M., 1995, AJ 110, 2027

\section{Appendix A: Kinematic profiles of the sample galaxies}

Shown here are the kinematic profiles of all sample galaxies with absorption lines strong enough for fourier fitting ${ }^{7}$. The profiles were obtained by applying the fourier fitting method with Gauss-Hermite polynomials on the sample spectra. The graphs plot rotational velocity, velocity dispersion, $h_{3}$ and $h_{4}$ values as a function of the distance from the centers of intensity. Filled and open circles refer to opposite sides from the centers. The spatial direction of the galaxy's side where the filled circles point to is given at the upper right corners of the plots. Distances from the centers are given in arcseconds below the plots and in units of the effective radii, when available (see Table 1 ), on top of the plots. The position angle of the slit was generally aligned with the optical major axis of the target galaxy, with the exceptions of NGC 2663 and NGC 4697, where two different position angles where used.

\footnotetext{
7 Only the first page of Fig. 6 is printed, the full figure is available at http://www.edpsciences.org
} 

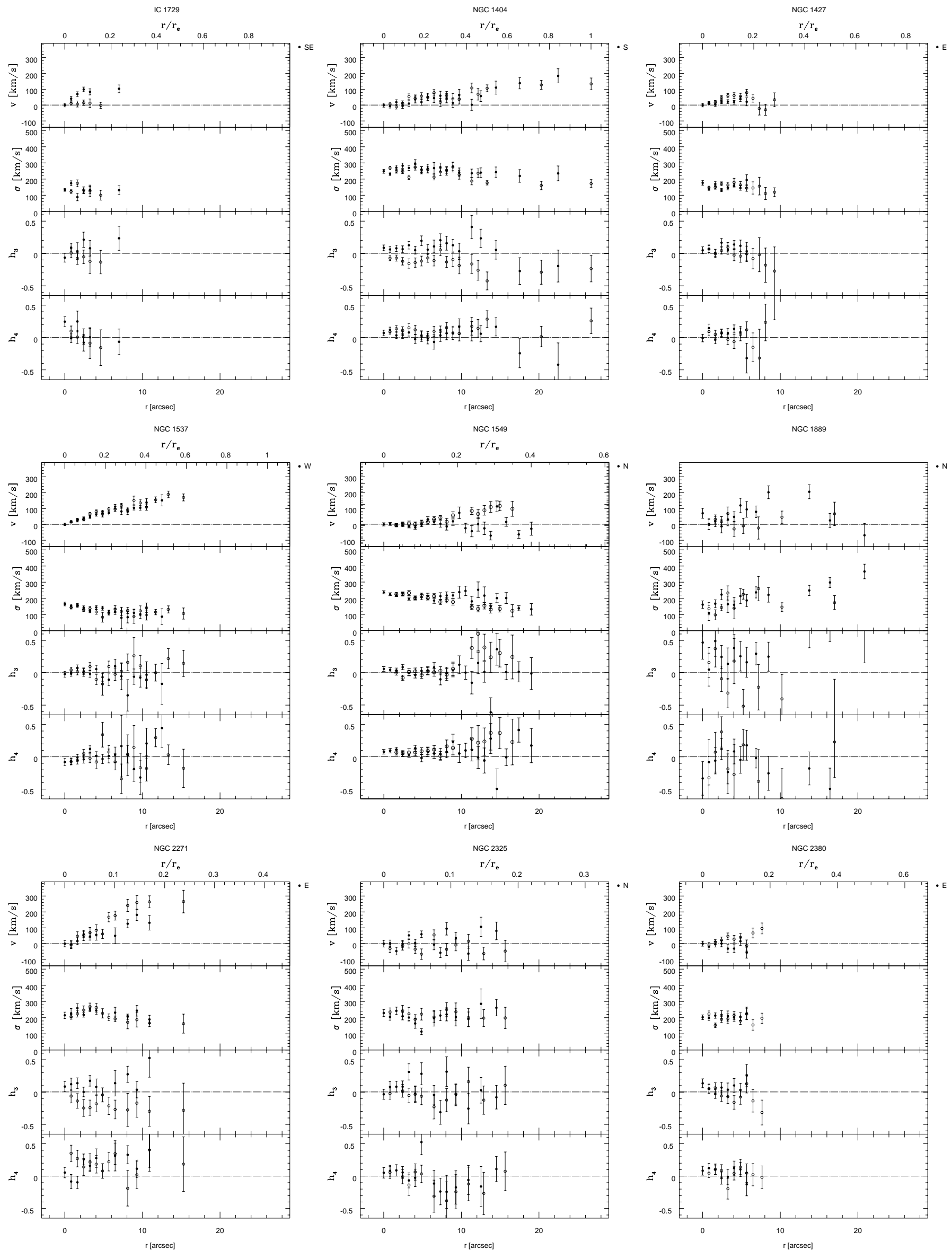

Fig. 6. 